\title{
UNIVERSITÄTSSCHULEN
}

DDS - Die Deutsche Schule

111. Jahrgang 2019, Heft 1, S. 8-21

https://doi.org/10.31244/dds.2019.01.02

(c) 2019 Waxmann

Eva Matthes

\section{Universitätsschulen in deutschen Staaten - historische Fallbeispiele}

\section{Zusammenfassung}

Die höhere Lehrerbildung war im 19. Jahrhundert als „Philologen“-Ausbildung konzipiert. Der Pädagogik wurde die Wissenschaftlichkeit abgesprochen, und die pädagogische Praxis sollte in klarer Trennung von der Universität gelernt werden. Herbart und die Herbartianer stellten sich gegen diese Auffassung und gründeten pädagogische Universitätsseminare mit angeschlossenen Übungsschulen. Diese sollten der theoriegeleiteten Ausbildung der Lehramtsstudierenden und der Weiterentwicklung der pädagogischen Wissenschaft dienen. Petersen modifizierte in den 1920er-Jahren den Charakter der Jenenser Universitätsübungsschule hin zur „Pädagogischen Tatsachenforschung“.

Schlüsselwörter: Pädagogisches Seminar, Theorie und Praxis, pädagogische Wissenschaft, höhere Lehrerbildung, Herbart und Herbartianer, Universitätsübungsschule, Peter Petersen, Versuchsschule, Pädagogische Tatsachenforschung

\section{University Training Schools in German States - Historical Case Studies}

\section{Summary}

In the $19^{\text {th }}$ century, the education of secondary school teachers [höhere Lehrerbildung] in Germany was conceptualized as education of "philologists." Pedagogy was denied a scientific basis, and the pedagogical practice was to be learnt clearly separated from the university. Herbart and the Herbartians opposed this view and founded pedagogical university seminars with affiliated training schools. These should serve both the theory-driven education of teacher students and the advancement of the pedagogical science. In the 1920s, Petersen modified the character of the training school at the University of Jena towards the so-called "Pedagogical Documentary Research" ["Pädagogische Tatsachenforschung"].

Keywords: pedagogical seminar, theory and practice, pedagogical science, secondary teacher education, Herbart and Herbartians, university training school, Peter 
Universitätsschulen in deutschen Staaten - historische Fallbeispiele |

Petersen, experimental school, "Pedagogical Documentary Research" [Pädagogische Tatsachenforschung]

\section{Einleitung}

Als erstes sind zentrale Begriffsklärungen nötig, die sich aus der Geschichte der Lehrerbildung ergeben. In den folgenden Fallbeispielen taucht der Begriff der Universitäts(übungs)schule (fast) immer im Zusammenhang mit dem des „Pädagogischen Seminars“, manchmal - etwa bei dem Herbartianer Ziller - mit dem des "Akademischen Pädagogischen Seminars" auf. Letzteres verlangt nach einer notwendigen begrifflichen Abgrenzung. Wenn im Folgenden von „Pädagogischen Seminaren“ gesprochen wird, sind nicht die Lehrerseminare für die - bis in das 20 . Jahrhundert hinein - nicht-akademische Volksschullehrerbildung gemeint, die durchaus auch mit Übungsschulen verbunden waren, aber eben keinerlei Bezüge zu den Universitäten aufwiesen. Die beiden Lehrergruppen - Volksschullehrer*innen und Lehrer*innen höherer Schulen - existierten im 19. Jahrhundert strikt getrennt und in klarer Abgrenzung voneinander.

Die Pädagogischen Seminare mit Universitätsübungsschulen sind im 19. Jahrhundert im Kontext der Bemühungen um eine pädagogische Ausbildung der angehenden höheren Lehrer als von den Theologen losgelöster eigenständiger Lehrerstand und um die universitäre Verankerung einer eigenständigen pädagogischen Wissenschaft als Berufswissenschaft jener - in Auseinandersetzung mit der im Examen pro facultate docendi (Preußen 1810) festgeschriebenen Dominanz der Philologie bzw. der Fachwissenschaften - zu sehen. Sie sind wiederum klar abzugrenzen von einigen im 18. und 19. Jahrhundert bestehenden „Gymnasial-Seminaren“, die von den Universitäten getrennt existierten und an denen angehende höhere Lehrer ihre praktischmethodische Ausbildung während der Zeit ihres universitären Studiums - das ist der Unterschied zum Studienseminar des Referendariats - erfahren sollten. 1826 führte Preußen nach Abschluss des universitären Studiums ein pädagogisches Probejahr unter Aufsicht des Schuldirektors ohne Prüfung ein, das vielfacher Kritik ausgesetzt war. 1890 wurde die zweite Ausbildungsphase eine feste Institution in Preußen, die zwei Jahre dauerte (erstes Jahr: „Seminarjahr“, zweites Jahr: „Probejahr“). Ausbilder waren hierfür ausgewählte Mitglieder des Lehrerkollegiums. In den anderen deutschen Ländern liefen die Entwicklungen strukturell ähnlich (vgl. Tenorth, 1987; Titze, 1991; Matthes, 2000).

Universitätsschulen waren im deutschsprachigen Raum somit die absoluten Ausnahmen. Das Fach Pädagogik hatte sich in dieser Zeit noch nicht an den Universitäten etablieren können. Herbart und vor allem die Herbartianer kämpften für beides - 
und waren hierbei mit großem Misstrauen ihrer Professorenkollegen konfrontiert (vgl. Prondczynski, 1998).

Bei der Skizzierung der einzelnen Universitätsschulen wird die zentrale Fragestellung dieses Beitrags sein, ob sie entweder eine stärkere Forschungsorientierung oder eine stärkere Ausbildungsorientierung aufweisen oder ob sich die Orientierungen gegebenenfalls miteinander verschränken. Am Ende des Beitrags wird hierzu ein erstes Resümee gezogen. ${ }^{1}$

\section{Universitätsübungsschulen Herbarts und der Herbartianer}

\subsection{Gemeinsame Grundanliegen}

Entscheidend für die Forderung Herbarts und der Herbartianer nach Einführung von Universitätsübungsschulen sind zentral drei Aspekte:

a) ihr Verständnis der Pädagogik als zentrale wissenschaftliche Grundlage des Lehrerberufs, nicht zuletzt auch des Berufs der höheren Lehrer; in diesem Kontext forderten sie die Etablierung eines eigenständigen höheren Lehrerstandes mit einem professionelle Identität stiftenden pädagogisch-didaktischen Reflexionswissen, grundgelegt von an den Universitäten einzurichtenden Professuren für Pädagogik;

b) ihre spezifische Thematisierung des Erfahrungsbegriffs: Erfahrung könne dann für eine professionelle pädagogische Praxis fruchtbar werden, wenn sie an Theorie angebunden werde, sich mit theoretischem Wissen verknüpfe; ansonsten erstarre sie zur Routine;

c) der Glaube an die Güte des eigenen pädagogisch-didaktischen Systems, das der Freiheit der praktischen Erprobung und Umsetzung sowie der Verbreitung durch die Absolventen der Übungsschulen bedürfe. Dem letzten Punkt ist die Vorstellung inhärent, dass sich die Theorie an der Praxis zu bewähren habe und tendenziell auch Modifikationen/Weiterentwicklungen des pädagogisch-didaktischen Systems durch Anregungen aus der Praxis erfolgen können; die zentralen Grundlagen des pädagogisch-didaktischen Systems - z. B. das Prinzip des „erziehenden Unterrichts“ - bleiben davon allerdings unberührt (vgl. Matthes, 2015); das Wissenschaftsverständnis Herbarts und der Herbartianer ist im Kern deduktiv (vgl. Matthes, 2015; Coriand, 2003; Wittenbruch, 1972).

Trotz grundlegender Übereinstimmungen zeigen sich in den einzelnen Konzeptionen und Ausgestaltungen doch auch klare Unterschiede. Diese sollen im Folgenden durch Einzelfallbeschreibungen deutlich gemacht werden.

1 Dieser Beitrag kann nur eine erste Hinführung zum Thema sein. Die Geschichte der Universitätsschulen in Theorie und Praxis muss erst noch geschrieben werden. 
Universitätsschulen in deutschen Staaten - historische Fallbeispiele |

\subsection{Konkretisierungen}

\subsubsection{Johann Friedrich Herbarts pädagogisches Seminar}

Johann Friedrich Herbart (1776-1841) wollte Hörern seiner pädagogischen Vorlesung an der Universität Königsberg die Möglichkeit eröffnen, die von ihm vorgetragenen Theorien in der Praxis zu überprüfen. Er wollte damit ihr pädagogisches Selbstverständnis stärken und die Professionalisierung angehender Lehrkräfte, auch für höhere Schulen, vorantreiben. Rein philologisch/fachwissenschaftlich ausgebildete Lehrkräfte hielt Herbart für absolut unzureichend, um einen erziehenden Unterricht auf hohem Niveau umzusetzen (vgl. Herbart, 1909a, S. 79). Das bestehende Schulsystem erschien ihm pädagogisch minderwertig, in besonderem Maße auf der Mittelstufe, deren Schüler besonderes pädagogisches Geschick erforderten (vgl. S. 79 f.).

Herbart bat den Kurator seiner Universität 1815 um die Erweiterung seines seit 1810 bestehenden didaktischen Instituts, das Hörern von ihm die Möglichkeit bot, ausgewählte Lehrstunden, die sie in ihrer Funktion als Hauslehrer mit ihren Schülern durchführten, an der Universität, vor Herbart und Kommilitonen, vorzuführen. Herbart kommentierte diese „Vorführung“ in einem anschließenden Vier-AugenGespräch (vgl. S. 31). Er hielt dieses didaktische Institut allerdings nicht für ausreichend; die ihm zentral erscheinende Verbindung von Unterricht und Erziehung - prägend waren für ihn seine Hauslehrererfahrungen gewesen - war in diesem didaktischen Institut wie auch in den überkommenen Schulen seines Erachtens nicht umsetzbar. Er beantragte deshalb die Einrichtung eines - von ihm geleiteten - pädagogischen Instituts/Seminars - er nannte es auch „Pädagogium“ (S. 80) -, das als eine Art kleine, familienähnliche Internatsschule konzipiert war. Es sollte Jungen im Alter von 9 bis 15 Jahren aufnehmen, maximal 20. „Das Pädagogium darf nicht zu groß seyn. Sonst würde es gehen wie in großen Schulen, wo das Schwungrad die Beweger mit sich fort reißt, so daß Routine entsteht anstatt der Erfahrung“ (ebd.). Wohlhabende Eltern, die einen großen Teil der anfallenden Kosten übernehmen könnten, sollten Knaben auf dieses Pädagogium schicken; der Staat hätte „für ein paar feste Lehrer zu sorgen, welche letztere jedoch niemals anders, als auf den Vorschlag des Directors auszusetzen wären, weil derselbe sonst unmöglich für die nöthige Einheit stehen könnte“ (ebd.). Bis zu zwei Drittel der Unterrichtsstunden wären von den Studenten zu übernehmen, die mit den Knaben zusammenlebten und sie deshalb genau kennten, was ihrem Unterricht eine gute Basis gebe (S. 81). „Der Zweck, sie dadurch im Lehren zu üben, fällt in die Augen“ (ebd.). Das „Pädagogische Seminar“ wurde verwirklicht; in einem Entwurf zu einem Reglement aus dem Jahr 1820 heißt es im $₫ 1$ : „Das pädagogische Seminar ist eine Anstalt für Studirende, worin sie die wichtigsten und schwersten Theile der Erziehungskunst durch Anschauung und eigene Übung sollen kennen lernen“ (1909b, S. 63). In einem Bericht über das 
pädagogische Seminar von Ostern 1821 bis Ostern 1823 zieht Herbart eine insgesamt positive Bilanz:

„Daß meine pädagogischen Vorlesungen in den letzten Jahren über Erwartung ${ }^{2}$ zahlreich besucht wurden, kann ich nur der Aufmerksamkeit und dem guten Glauben zuschreiben, welchen [sic!] das Seminar bewirkte. Die wöchentlichen Versammlungen Sonnabends, wo während anderthalb Stunden bald von diesem bald von jenem Lehrer Proben des Unterrichts gegeben werden, sind fortdauernd im Gange“" (1909a, S. 199).

Eindeutig ist bei Herbart die Vorstellung, dass sein eigenes pädagogisch-didaktisches System erprobt werden sollte - allerdings nicht in enger Anleitung: „Meine Seminaristen bekommen von mir fast gar keine directen Vorschriften; sie haben Pädagogik gehört, und wissen also was ich will“ (S. 200).

Ein zentraler Punkt für ihn ist der theoriebezogene Austausch über die praktischen Erfahrungen. In seinem ersten Entwurf zur Anlage eines Pädagogischen Seminars aus dem Jahr 1809 formuliert Herbart:

„Als ein vorzüglich wichtiges Resultat der ganzen Einrichtung wäre die Summe von Beobachtungen anzusehen, welche hier regelmäßiger als sonst gesammelt und verständiger als sonst aufgefaßt, gedeutet und auf die allgemeine Theorie zu deren Ergänzung bezogen werden würden. Es wäre daher ein Hauptgeschäft der Erzieher [der Studenten; E.M.], von Zeit zu Zeit praktisch-pädagogische Aufsätze zu entwerfen, in welchen sie der Theorie von Seiten der Erfahrung entgegenkämen“ (S. 24 f.).

Mit der Rückkehr Herbarts nach Göttingen im Jahr 1833 endete das pädagogische Seminar in Königsberg.

\subsubsection{Heinrich Gustav Brzoskas Konzeption eines pädagogischen Seminars mit Übungsschulen}

Herbarts Schüler Heinrich Gustav Brzoska (1807-1839), der Herbarts erster Assistent an seinem pädagogischen Seminar an der Universität Königsberg gewesen war, bemühte sich als Privatdozent und später außerordentlicher Professor für Philosophie an der Universität Jena um die Einrichtung eines pädagogischen Seminars mit unter der Leitung des Seminardirektors angeschlossenen Schulen - ein sehr weitgehender, anspruchsvoller Plan. Auch wenn es bei der Konzeption blieb, ist diese, dargelegt in der 1836 erschienenen Schrift Die Nothwendigkeit pädagogischer Seminare auf

2 Der Besuch der pädagogischen Vorlesung war freiwillig. Pädagogik war kein Bestandteil der universitären höheren Lehramtsausbildung; die angehenden höheren Lehrer mussten auch kein pädagogisches Examen absolvieren (vgl. Herbart, 1909a, S. 199). 
der Universität und ihre zweckmäßige Einrichtung, so grundlegend, dass hier kurz darauf eingegangen werden soll. Das pädagogische Seminar soll nach Brzoska der Ort sein, an dem diejenigen, die später in pädagogischen Kontexten tätig sein werden, sich intensiv, in enger Verzahnung von Theorie und Praxis, mit pädagogischen Fragestellungen beschäftigen. Es gehe hierbei nicht um Methodenausbildung oder das Erlernen von Techniken; vielmehr müsse das pädagogische Seminar eine Anstalt sein, „in welcher junge Männer, die sich dem Erziehungsgeschäft widmen wollen, unter der Leitung eines hinlänglich dazu geeigneten Mannes theoretisch und praktisch nicht nur lernend, sondern selbständig forschend und vielseitig sich übend“, sich auf ihren Beruf vorbereiten könnten (1836, S. $188 \mathrm{f}$; Hervorh. i. O.). Der Gedanke des forschenden Lernens in der Lehramtsausbildung ist hiermit grundgelegt. Das Studium am pädagogischen Seminar solle so eingerichtet werden, dass „das freie Selbstforschen so viel als möglich begünstigt wird und daß sie [die Studenten; E. M.] alles durch Bearbeitung in individueller Weise zu ihrem wahren Eigentume machen können“; neben einer „umfassenden theoretischen Bildung“ sollen sie eine „vollkommen hinreichende Veranlassung und Gelegenheit zu pädagogischen Beobachtungen und zu praktischen Übungen“ erhalten (S. 317).

\subsubsection{Karl Volkmar Stoys pädagogisches Universitäts-Seminar mit Übungsschule}

Karl Volkmar Stoy (1815-1885), auch noch unmittelbarer Schüler Herbarts, kam 1843 an die Universität Jena. Er strebte ebenfalls danach, ein pädagogisches Seminar mit Übungsschule einzurichten, und war damit - nicht zuletzt dank der Vorarbeiten Brzoskas und bescheidenerer Vorstellungen bezüglich der Schule - erfolgreich. 1844 konnten Übungsklassen eröffnet werden; zugestanden wurden ihm seitens der Stadt Jena zunächst eine Oberklasse, die von 30 Mädchen besucht wurde, und eine Unterklasse mit 33 Schülerinnen der Frei- bzw. Armenschule - die Zusammensetzung der Schülerschaft war somit eine deutlich andere als die des Herbartschen Pädagogiums. 1848 übergab die Stadt Jena Stoy die gesamte dreiklassige zweite Knabenschule als Übungsschule. Nach einer Unterbrechung durch den Weggang Stoys nach Heidelberg von 1866 bis 1874 konnte er ab 1876 die gesamte koedukative Volks- und Armenschule Jenas übernehmen, die in fünf Klassen 181 Kinder, darunter 50 Mädchen, zählte. Unterricht und umfangreiches Schulleben wurden verantwortet von einem Oberlehrer, drei Klassenlehrern und 28 Seminaristen; die Oberaufsicht hatte Stoy als Direktor, der allerdings der Stadt, da es sich ja um eine städtische Schule handelte, rechenschaftspflichtig war (vgl. Coriand, 2000, S. 123 ff.).

3 Wilhelm Rein maß dieser Schrift für die Entstehung von pädagogischen Universitäts-Seminaren mit Übungsschulen eine solch große theoretische Bedeutung und praktische Wirksamkeit bei, dass er sie 1887 mit einem ausführlichen Vorwort erneut auflegte. 
Als zentrale Begründung für die Tätigkeit der angehenden Lehrer in der einem pädagogischen Seminar angeschlossenen Übungsschule führt er aus:

„Da die Aneignung einer durch die Einsicht geregelten Praxis nur allmälig durch eine zusammenhängende Reihe von wohl vorbereiteten und wohl verarbeiteten Erfahrungen bewirkt werden kann, so muß den künftigen Lehrern Gelegenheit zur Ertheilung nicht etwa von bloßen Probelektionen, sondern von zusammenhängendem Unterrichte und zum Umgange mit Schülern gegeben werden“ (1876, S. 184; Hervorh. i. O.).

Entscheidend ist hierbei, dass die Erfahrungen durch pädagogische Theorie, den Aufbau eines „pädagogischen Gedankenkreises“ (Stoy, 1869, S. 43), vorbereitet und somit - sozusagen theorieaffin - verarbeitet werden können. Auch Stoy versteht hierbei - wie Herbart - pädagogische Theorie nicht als Rezeptologie. Als entscheidend sieht er die Ausbildung einer pädagogischen Grundhaltung auf der Basis der Herbartschen Pädagogik (in der Auslegung Stoys) an. Allerdings klingen einige Ausführungen von ihm doch recht dogmatisch:

„Letztes Ziel der pädagogischen Bildung im Seminar ist möglichste Fertigkeit und Sicherheit in der Handhabung der pädagogischen Gesetze und in Beobachtung der im einzelnen Falle nöthigen pädagogischen Rücksichten“ (ebd.),

wobei der geforderte Bezug auf die „Handhabung der pädagogischen Gesetze“ jedoch durch die als notwendig erachtete individuelle Konkretisierung derselben wieder relativiert wird. Auffällig ist allerdings, dass für Stoy die „pädagogischen Gesetze“ bereits klar feststehende, nicht mehr zu verändernde zu sein scheinen; deshalb findet sich auch bei ihm nicht der Gedanke der Anreicherung/Modifikation der wissenschaftlichen Theorie durch die praktischen Erfahrungen an der Übungsschule.

Die Seminaristen sollen die Übungsschule dazu nutzen, die Theorie anzuwenden, um dabei zu lernen, „sich selbst [zu] regieren in dem Verkehr mit den Schülern“ (Stoy, 1884; zit. in: Bliedner, 1886, S. 328).

Die Vorgehensweise für die Seminaristen an der Übungsschule war folgende: Sie erhielten durch den Direktor Anleitung zum Unterricht in einer Klasse, sie arbeiteten ein Programm ihres Unterrichts aus und legten dasselbe dem Direktor zur Beurteilung vor; beim Unterricht der Seminaristen waren der Direktor und andere Seminaristen anwesend, es wurde Protokoll geführt (vgl. ebd.). „Später werden die Leistungen der jungen Lehrer in besonderen Konferenzen sorgfältig und gründlich beurteilt nach den Gesetzen der Theorie durch die Zuhörer und den Direktor“ (ebd.). Dies verlief folgendermaßen: Zuerst kam der Seminarist, auch „Praktikant“ genannt, der die Stunde gehalten hatte, zu Wort, um seine schriftliche Selbstkritik zu verlesen. Nach ihm trug ein vor der Übungsstunde benannter „Recensent“ die ebenfalls 
schriftlich vorbereitete Kritik vor. Dann erfolgte eine Diskussion, an der sich alle Hospitanten beteiligen konnten. Das Schlusswort kam dem Seminardirektor - Stoy - zu. Auch das „Criticum“ (Bliedner, 1886, S. 176) wurde sehr genau protokolliert. Darüber hinaus fanden regelmäßig Schulkonferenzen, „Scholastiken“, statt, in denen Erziehungsfragen und die individuelle Entwicklung einzelner Schüler*innen gemeinsam besprochen wurden (ebd., S. 177f.).

„Auf diese Weise lernt jeder Seminarist andere beurteilen, aber auch sich selbst, und so verwandelt er die Theorie in succum und sanguinem [so geht diese ihm ,in Fleisch und Blut“ über; E. M.]“ (Stoy, 1884; zit. in: Bliedner, 1886, S. 328 f.).

Wichtig ist für Stoy, dass die Unterrichtsbeobachtungen der Praktikanten nicht willkürlich erfolgen; er plädiert vielmehr für eine „auf der Unterlage sorgfältiger Vorbereitungen und durch planmässige Reflexionen wohlgeleitete [ ] Beobachtung" (Stoy, 1878, S. 421).

\subsection{Wilhelm Reins pädagogisches Universitäts-Seminar mit Übungsschule}

Wilhelm Rein (1847-1929), Schüler Stoys und Zillers, wurde nach Stoys Tod 1885 sein Nachfolger an der Universität Jena, und mit der Übernahme seiner Professur bemühte er sich - in Abgrenzung von der Übungsschule Stoys - um die Einrichtung einer von der Stadt Jena unabhängigen Übungsschule, die der Universität angegliedert wurde, was ihm auch gelang. 1886/87 eröffnete er sein pädagogisches Universitätsseminar mit Übungsschule. Diese umfasste nur drei Klassen mit je maximal 20 Kindern, und zwar beginnend mit dem ersten, dritten und fünften Schuljahr; obwohl die Schule immer nur drei Klassen führte, war sie eine achtklassige Schule, d.h., jedes Kind konnte hier seine Schulzeit beenden.

Die Aufgabe des pädagogischen Universitätsseminars mit Übungsschule bestimmt Rein als eine doppelte: „Einerseits will es der Fortentwicklung der pädagogischen Wissenschaft, andererseits der theoretischen und praktischen Ausbildung wissenschaftlich strebsamer Erzieher dienen“" (Rein, 1911, S. 315) ${ }^{5}$. Beachtenswert ist, dass

4 Als Beispiel für ein Kritikum, eine Selbstkritik und eine Rezension siehe etwa Bliedner, 1886, S. $306 \mathrm{ff}$.

5 Die Formulierung ist (ohne entsprechenden Verweis) nahezu identisch mit der Tuiskon Zillers (1817-1882), der ja ebenfalls ein pädagogisches Universitätsseminar mit Übungsschule an seiner Wirkungsstätte, der Universität Leipzig, eingerichtet hatte; interessant sind allerdings die kleinen Unterschiede: Bei Ziller heißt es: „Das akademisch-pädagogische Seminar hat einerseits der Fortentwickelung der pädagogischen Wissenschaft, andererseits der praktischen Ausbildung wissenschaftlich strebsamer Lehrer zu dienen" (Bergner, 1886, S. 1; Hervorh. i.O.). Rein betont wie Ziller die von Stoy deutlich weniger als notwendig angesehene Fortentwicklung der pädagogischen Wissenschaft durch Seminar und Übungsschule und nennt darüber hinaus neben der praktischen auch die pädagogisch-theoretische Ausbildung der angehenden Erzieher; indem er zudem von „Erziehern“ spricht, macht er seinen volks- 
er „die Fortentwicklung der pädagogischen Wissenschaft“ an erster Stelle nennt und im selben Text auch davon spricht, „die heranwachsende Generation der Erzieher in die wissenschaftliche Werkstätte einzuführen“ (ebd.). Er hebt die unverzichtbare Verbindung zwischen Theorie und Praxis hervor. Auch für ihn ist die Herbartsche Pädagogik grundlegend, die jedoch nicht sklavisch nachgeahmt werden solle, sondern vielmehr der Fortentwicklung bedürfe (vgl. Rein, 1888, S. 6 f.). Er betont die hierfür hilfreiche Freiheit der universitätseigenen Übungsschule, denn diese könne „ungehindert mancherlei Versuche anstellen, selbstverständlich nicht plan- und zwecklos, sondern solche, die durch theoretische Überlegungen gut vorbereitet sind: z. B. Änderungen im Lehrplan, Einführung neuer Lehrmittel, Prüfung neuer Lehrbücher, psychologische und physiologische Untersuchungen, neue Verfahrungsweisen der allgemeinen und speziellen Methodik usw." (Rein, 1911, S. 318). In der Theorie Verankertes und in der praktischen Erfahrung Bewährtes könnte dann auch in den Staatsschulen übernommen werden (vgl. ebd.).

Der Aufbau des pädagogischen Seminars mit Übungsschule war ganz ähnlich wie bei Stoy: Es gab das „Theoretikum“ mit der theoriegeleiteten Diskussion pädagogisch-didaktischer und methodologischer Fragestellungen, das „Praktikum“, die Probelektion in Gegenwart des Direktors und aller Seminarmitglieder, mit der von Stoy bereits bekannten nachfolgenden Selbstbeurteilung, und die während der Stunde angefertigte Rezension. Hervorzuheben ist, dass für die Stunde ein „Frageprotokollant“ bestimmt wurde, der „während des Praktikums über die Anzahl der an die einzelnen Schüler gerichteten und richtig, falsch oder gar nicht beantworteten Fragen, sowie der von den Schülern gegebenen Zusammenfassungen ein Verzeichnis anzulegen“ hatte (S. 322) - hier kann man Anfänge eines forschenden Lernens schon klar erkennen. In der wöchentlich einmal stattfindenden „Konferenz“ wurden allgemeine Schulangelegenheiten und besonders das wöchentliche Praktikum besprochen (vgl. hierzu insgesamt S. $321 \mathrm{f}.)^{6}$

\section{Peter Petersens Erziehungswissenschaftliche Anstalt und die Jena-Plan-Schule als Universitätsschule}

Peter Petersen (1884-1952), ${ }^{7}$ der 1923 auf die ordentliche Professur für Pädagogik der Universität Jena berufen wurde, inszenierte einen Bruch, einen absoluten Neuanfang gegenüber dem Pädagogischen Seminar und der Übungsschule Wilhelm Reins.

erzieherischen Anspruch deutlich; das pädagogische Seminar mit Übungsschule soll allen Erziehern unterschiedlichster Schulen und pädagogischer Einrichtungen zur Verfügung stehen (vgl. Rein, 1911, S. 317).

6 Die Begrifflichkeiten Reins - Theoretikum, Praktikum und Konferenz - sind identisch mit denen Zillers (vgl. Bergner, 1886, S. 5 f.).

7 Es ist hier nicht der Ort, ausführlich auf Petersens problematische Rolle in der NS-Zeit einzugehen; einen guten Überblick zum aktuellen Diskussionsstand bieten Fauser, John \& Stutz, 2012 (v. a. Teil II: Die NS-Zeit). 
Hierzu nur einige Hinweise: Er kritisierte die Reinsche Übungsschule sehr scharf als Lern- und Drillschule (vgl. Petersen \& Wolff, 1925, S. 2). Die in dieser stattfindende Lehrerausbildung sah er als Handwerksausbildung an (vgl. ebd.). Rein und frühere Mitglieder seines Pädagogischen Seminars und der Übungsschule widersprachen nachdrücklich und empört dieser Darstellung, sowohl was die Gestaltung der Schule als auch was die theoretische und praktische Ausbildung der Praktikanten betraf.

„[...] mit besonderem Dank haben wir es allezeit empfunden, daß wir auch Mittel und Wege theoretisch und praktisch hatten prüfen lernen, dem in der Wirklichkeit der öffentlichen Schule Geltung und Eingang zu verschaffen, was wir als richtig erkannt hatten. Wer sich davon überzeugen will, lese die Seminarberichte und die zahlreichen Abhandlungen und Schriften der früheren Seminarmitglieder nach“ (Rein, 1925, S. 11).

Petersen verlangte zunächst die Auflösung der Reinschen Übungsschule, als er Gegenwind erfuhr, deren Umwandlung, und betonte in diesem Kontext, dass die Übungsschule „als eine Stätte freier schulischer Versuche zu erhalten sei“ (zit. nach John, 2012, S. 111). Am 14. Mai 1924 eröffnete Petersen seine „Erziehungswissenschaftliche Anstalt der Thüringischen Landesuniversität“ mit der „freien Versuchsschule“ (ebd., S. 113). Die Mitglieder der Erziehungswissenschaftlichen Anstalt waren Aspiranten für das Volksschul- wie für das höhere Lehramt sowie Lehrkräfte, die sich fortbilden und/oder promovieren wollten (vgl. ebd., S. 114). Die Versuchsschule zählte 192669 Kinder in drei geleiteten Gruppen (1.-3., 4.-6., 7./8. Schuljahr) ${ }^{8}$ (vgl. ebd., S. 117 f.). Sie war als „wissenschaftliche Versuchsschule“ der Universität Jena angegliedert und dem Lehrstuhlinhaber für Pädagogik, also Petersen, unterstellt (vgl. ebd., S. 120). 1926 beantragte dieser beim Ministerium, den Namen „UniversitätsÜbungsschule“ in „Universitätsschule Jena“ zu ändern. Hier wird wieder seine immense Abgrenzung von der Reinschen Übungsschule deutlich: Petersen betont, dass seine Schule keine bloße „Übungsschule zur Erlernung von Unterrichtstechniken und -methoden“ mehr sei - das war die Übungsschule allerdings auch im Verständnis Reins und seiner Mitstreiter nicht; vielmehr verstanden sie sie durchaus als Übungsfeld für eine reformierte Erziehung -, dass jene vielmehr „der Forschung und der pädagogischen Anschauung für die Studierenden der Erziehungswissenschaft“ diene (zit. n. John, 2012, S. 119; vgl. hierzu auch Döpp, 2003, S. 127 f.). Dem Antrag auf Namensänderung wurde zum 1. Juli 1926 stattgegeben, und Petersen beschreibt in

8 Als Hintergrund ist hierfür wichtig, dass Thüringen als erstes Land in der Weimarer Republik die Lehrerbildung in seinem Lehrerbildungsgesetz vom 8. Juli 1922 neu regelte. Es schrieb nach einer Übergangszeit bis 1927 für alle Lehrkräfte öffentlicher Schulen Abitur, Universitätsstudium und „praktisch-pädagogische Schulung“ vor und übertrug die Lehrerbildung der Landesuniversität Jena. Die bisherigen Volksschul-Lehrerseminare sollten bis 1927 abgebaut werden; bis zu diesem Zeitpunkt sollte also das Ziel der Akademisierung der Volksschullehrerausbildung erreicht sein (vgl. John, 2012, S. 99 f.). Allerdings eröffnete bereits 1928 ein „Pädagogisches Institut“, das in Kooperation mit der Universität den Kern der Volksschullehrerausbildung ausmachte und in direkte Konkurrenz zur Erziehungswissenschaftlichen Anstalt Peter Petersens trat (vgl. Retter, 1996, S. 18). 
einer Darstellung der verschiedenen Elemente der Lehrerbildung an der Universität Jena die Aufgabe der Universitätsschule folgendermaßen: Sie sei „freie Versuchsschule zur Durchführung und Erprobung neuer Methoden und Erziehungsprobleme aller Art, für jugendkundliche Untersuchungen“. Deutlich kommt auch hier wieder seine Abgrenzung von der vorangegangenen Universitätsübungsschule zum Tragen, wenn er fortfährt:

„Nicht im Sinne der alten Übungsschule, die [in seiner Sichtweise; E. M.] mit Musterlektionen arbeitete und die Studenten hospitieren ließ, um die Muster aufzunehmen und zu lernen, es ebenso zu machen, sich auch einmal in dieser Schule zu erproben [...]“ (Petersen, 1926, S. 53).

Seit Petersens Vorstellung seiner Reformschule auf der IV. Internationalen Konferenz des „Weltbundes für Erneuerung der Erziehung“ in Locarno 1927 ging die Universitätsschule der Landesuniversität Thüringen als "Jena-Plan-Schule“ in die Geschichte ein. ${ }^{9}$

Wenngleich der Bruch zwischen der Universitätsübungsschule Reins und der Universitätsschule Petersens nicht so groß war, wie er selbst suggerierte - sich generell viel mehr Kontinuitäten zwischen Herbartianismus und Reformpädagogik/ „Neuer Erziehung“ zeigen, als über einen langen Zeitraum in der pädagogischen Historiographie postuliert wurde -, so wurde die Universitätsschule doch in einem ganz anderen Ausmaß und vor allem in einer vorher nicht dagewesenen methodischen Ausdifferenzierung zu einem Ort der „Pädagogischen Tatsachenforschung“. Im Zentrum standen genau angeleitete, schriftlich festgehaltene Beobachtungen, unterschieden nach „Einzelaufnahme“, „Lehreraufnahme“ und „Gesamtaufnahme“; diese wurden ebenfalls nach genauer Anleitung „deskriptiv“, „phänomenologisch“, „numerisch“, „zeichnerisch“, „statisch“ und „dynamisch“ sowie „kausal“ ausgewertet (MüllerPetersen, 1951; vgl. auch Petersen \& Petersen, 1965). Die Forschungsorientierung wurde also ganz klar ausgebaut (vgl. hierzu auch Kleinespel, 1998, S. 50-74).

Dass die Universitätsschule zunehmend ihre Ausbildungsfunktion für die Lehramtsstudierenden verlor, war allerdings nicht im Sinne Petersens, sondern der Einführung des „Pädagogischen Instituts“ (vgl. Fußn. 8) zuzuschreiben. Die Universitätsschule wurde somit nur noch zur praktischen Übungsstätte für solche Studierende, die sich freiwillig dazu bereitfanden; das waren im Wesentlichen Petersens Doktoranden und einige wenige pädagogisch besonders interessierte Studierende (vgl. Retter, 1996, S. 18).

9 Zu ihrer weiteren Entwicklung vgl. die instruktiven Beiträge in Fauser et al. (2012). 
Universitätsschulen in deutschen Staaten - historische Fallbeispiele |

\section{Schluss}

Die Pädagogik als Wissenschaft konnte sich im Zuge der Pädagogischen Konferenz vom Mai 1917 in der Weimarer Republik endlich an den Universitäten etablieren; das vorherrschende Paradigma wurde die „geisteswissenschaftliche Pädagogik“. Deren Repräsentanten lehnten - anders als etwa „experimentelle Pädagogen“ eine Universitätsschule - die jene sich angesichts ihrer empirischen Abstinenz nur als „Übungsschule“, als Ausbildungsstätte, denken konnten - mit Nachdruck ab. Ihre entscheidenden Argumente waren, dass die Einrichtung von Übungsschulen in Verbindung mit pädagogischen Seminaren zum einen der Pädagogik als (Geistes-) Wissenschaft sozusagen ihre wissenschaftliche Dignität und damit auch ihre Anerkennung unter den anderen, sich auf zweckfreie Wissenschaft fokussierenden Geisteswissenschaften der Philosophischen Fakultät raube und zum anderen den Studierenden suggeriere, dass die Pädagogik nicht eine Kulturhandlung, sondern eine Technik sei, die sie an der Universität in Verbindung mit einer Übungsschule erlernen könnten (vgl. etwa Spranger, 1925; Nohl, 1929; Matthes, 2009). ${ }^{10}$ Damit war das weitgehende Ende der Universitätsschulen - Peter Petersens Einrichtung überlebte als Spezifikum der Jenenser Universität in der SBZ/DDR noch bis 1950 - zunächst einmal besiegelt. ${ }^{11}$

Für die Gegenwart ist aus den historischen Fallbeispielen m.E. der Schluss zu ziehen, dass es wenig Sinn macht, Ausbildungs- und Forschungsintention von Universitätsschulen klar voneinander abzugrenzen oder gar gegeneinander auszuspielen, sondern dass es vielmehr notwendig ist, diese in eine fruchtbare Verbindung zu bringen und damit bei allen angehenden Lehrkräften einen forschenden Habitus, die Bereitschaft, fremden und eigenen Unterricht theoriefundiert und methodisch transparent $\mathrm{zu}$ analysieren, grundzulegen. Der mit bisherigen Universitätsschulen verbundene hohe Anspruch, einer „neuen Erziehung“, einer gegenüber der bestehenden grundlegend reformierten Schule den Weg zu bereiten und diese theoretisch abzusichern - den ja nicht nur Petersen, sondern auch Herbart und die Herbartianer vertraten -, wird m. E. hierbei tendenziell eher aufzugeben, zumindest deutlich niedriger zu hängen sein als in den geschichtlichen Beispielen.

10 Interessant ist, dass Spranger auch in der Konzeptualisierung seiner „Bildnerhochschule“ (Spranger, 1920, S. 40; Hervorh. i. O.) für die - in seinem Verständnis vom höheren Lehramt entschieden abzugrenzende Ausbildung der Volksschullehrkräfte - eine „offizielle Übungsschule" (S. 54) klar ablehnt. Er argumentiert hier damit, dass diese „immer die Gefahr“ enthalte, „Lehrer, Kinder und Methoden schematisch zu behandeln“ (ebd.). Zudem: „Die Schüler einer solchen Anstalt mit 300 unfertigen Lehrern würden sich auch nicht gerade zum Besten befinden" (ebd.). Spitzen gegen den Herbartianismus und dessen Übungsschulen sind in diesen Ausführungen unverkennbar.

11 Die 1974 gegründete „Versuchsschule des Landes Nordrhein-Westfalen und Wissenschaftliche Einrichtung der Universität“, kurz: „Bielefelder Laborschule“, war lange Zeit singulär (vgl. hierzu den einschlägigen Beitrag in diesem Heft). Erst in jüngster Zeit sind einige neue Universitätsschulen gegründet worden (vgl. die Berichte in diesem Heft). 


\section{Literatur}

\section{Primärliteratur}

Bergner, M. (Hrsg.). (1886). Materialien zur speziellen Pädagogik von Tuiskon Ziller des „Leipziger Seminarbuches“ (3., verm. Aufl.). Dresden: Bleyl \& Kämmerer.

Bliedner, A. (1886). Karl Volkmar Stoy und das pädagogische Universitätsseminar. Leipzig: Reichardt. [Mit Originaltexten Stoys aus den Akten des Seminars.]

Brzoska, H.G. (1836). Die Nothwendigkeit pädagogischer Seminare auf der Universität und ihre zweckmäßige Einrichtung. Leipzig: Barth. Neu hrsg. von W. Rein 1887 [Die Notwendigkeit ....].

Herbart, J. F. (1909a). Joh. Fr. Herbarts Sämtliche Werke in chronologischer Reihenfolge. Hrsg. v. K. Kehrbach \& O. Flügel. Bd. 14, hrsg. v. O. Flügel. Langensalza: Beyer \& Söhne.

Herbart, J. F. (1909b). Joh. Fr. Herbarts Sämtliche Werke in chronologischer Reihenfolge. Hrsg. v. K. Kehrbach \& O. Flügel. Bd. 15, hrsg. v. O. Flügel. Langensalza: Beyer \& Söhne.

Müller-Petersen, E. (1951). Kleine Anleitung zur Pädagogischen Tatsachenforschung und ihrer Verwendung. Marburg: Simons.

Nohl, H. (1929). Die Ausbildung der wissenschaftlichen Lehrer durch die Universität [1927]. In H. Nohl, Pädagogische Aufsätze (2., verm. Aufl.) (S. 183-189). Langensalza: Beltz.

Petersen, P. (1926). Die akademische Lehrerbildung an der Universität Jena. Schulreform, 5, 51-58.

Petersen P., \& Petersen, E. (1965). Die Pädagogische Tatsachenforschung. Paderborn: Schöningh.

Petersen, P., \& Wolff, H. (Hrsg.). (1925). Eine Grundschule nach den Grundsätzen der Arbeits- und Lebensgemeinschaftsschule. Weimar: Böhlau.

Rein, W. (1888). Aus dem Pädagogischen Universitäts-Seminar zu Jena. Langensalza: Beyer \& Söhne.

Rein, W. (1911). Pädagogik in systematischer Darstellung, Bd. 2 (2. Aufl.). Langensalza: Beyer \& Söhne.

Rein, W. (1925). Herr Professor Petersen in Jena und sein Angriff auf die Übungsschule des Pädag. Universitäts-Seminars 1886-1923. Jena: o. V.

Spranger, E. (1920). Gedanken über Lehrerbildung. Leipzig: Quelle \& Meyer.

Spranger, E. (1925). Die Ausbildung der höheren Lehrer an der Universität. Denkschrift der Philosophischen Fakultät der Friedrich-Wilhelms-Universität Berlin. Leipzig: Quelle \& Meyer.

Stoy, K.V. (1869). Organisation des Lehrerseminars: Ein Beitrag zur Methodologie der Pädagogik. Leipzig: Engelmann.

Stoy, K. V. (1876). Sechszehn [sic!] Thesen über die Pädagogische Bildung für das höhere Lehramt. Allgemeine Schul-Zeitung für das gesammte Unterrichtswesen, 53, 183-184.

Stoy, K. V. (1878). Encyklopädie, Methodologie und Literatur der Pädagogik (2., umgearb. u. verm. Aufl.). Leipzig: Engelmann.

\section{Sekundärliteratur}

Coriand, R. (2000). Karl Volkmar Stoy und die Idee der Pädagogischen Bildung. Würzburg: Ergon.

Coriand, R. (2003). Die Universitätsschulen im Lichte eines sich wandelnden TheoriePraxis-Verständnisses. In R. Coriand (Hrsg.), Herbartianische Konzepte der Lehrerbildung. Geschichte oder Herausforderung? (S. 79-99). Bad Heilbrunn: Klinkhardt. 
Döpp, R. (2003). Jenaplan-Pädagogik im Nationalsozialismus. Ein Beitrag zum Ende der Eindeutigkeit. Münster: Liт.

Fauser, P., John, J., \& Stutz, R., unter Mitwirkung von C. Faludi (Hrsg.). (2012). Peter Petersen und die Jenaplan-Pädagogik. Historische und aktuelle Perspektiven. Stuttgart: Steiner.

John, J. (2012). „Eine Schule - Ein Lehrerstand“. Lehrerbildung, Erziehungswissenschaftliche Anstalt und Universitätsschule als Peter Petersens Jenaer Handlungsfelder 1923 bis 1933. In P. Fauser, J. John \& R. Stutz unter Mitwirkung von C. Faludi (Hrsg.), Peter Petersen und die Jenaplan-Pädagogik. Historische und aktuelle Perspektiven (S. 77-160). Stuttgart: Steiner.

Kleinespel, K. (1998). Schulpädagogik als Experiment. Der Beitrag der Versuchsschulen in Jena, Chicago und Bielefeld zur pädagogischen Entwicklung der Schule. Weinheim \& Basel: Beltz.

Matthes, E. (2000). Theologe - Philologe - Fachwissenschaftler - Pädagoge? Historischsystematische Akzentsetzungen zur (Aus-)Bildung der Lehrer an höheren Schulen. Anregung. Zeitschrift für Gymnasialpädagogik, 46, 328-341.

Matthes, E. (2009). Die Pädagogik konstituiert sich als universitäres Fach. In U. Sandfuchs, J.-W. Link \& A. Klinkhardt (Hrsg.), Verlag Julius Klinkhardt 1834-2009. Verlegerisches Handeln zwischen Pädagogik, Politik und Ökonomie (S. 81-94). Bad Heilbrunn: Klinkhardt.

Matthes, E. (2015). Nicht-erwünschtes Wissen. Der Einsatz der Herbartianer für die Etablierung der Pädagogik an den Universitäten. In M. Häberlein, S. Paulus \& G. Weber (Hrsg.), Geschichte(n) des Wissens (S. 655-669). Augsburg: Wißner.

Prondczynsky, A. von (1998). Die Position Wilhelm Reins in den Diskussionen um die Einrichtung pädagogischer Universitätsprofessuren. In R. Coriand \& M. Winkler (Hrsg.), Der Herbartianismus - die vergessene Wissenschaftsgeschichte (S. 261-280). Weinheim: Deutscher Studien Verlag.

Retter, H. (Hrsg.). (1996). Peter Petersen und der Jenaplan. Von der Weimarer Republik bis zur Nachkriegszeit. Berichte - Briefe - Dokumente. Weinheim: Deutscher Studien Verlag.

Tenorth, H.-E. (1987). Lehrerberuf und Lehrerbildung. In K.-E. Jeismann \& P. Lundgreen (Hrsg.), Handbuch der deutschen Bildungsgeschichte, Bd. III: 1800-1870 (S. 250-270). München: Beck.

Titze, H. (1991). Lehrerbildung und Professionalisierung. In C. Berg (Hrsg.), Handbuch der deutschen Bildungsgeschichte, Bd. IV: 1870-1918 (S. 345-370). München: Beck.

Wittenbruch, W. (1972). Die Pädagogik Wilhelm Reins. Eine Untersuchung zum Spätherbartianismus. Ratingen: Henn.

Eva Matthes, Prof. Dr., geb. 1962, Lehrstuhlinhaberin für Pädagogik an der Universität Augsburg.

Anschrift: Universität Augsburg, Philosphisch-Sozialwissenschaftliche Fakultät, Lehrstuhl für Pädagogik, Universitätsstr. 10, 86159 Augsburg

E-Mail: eva.matthes@phil.uni-augsburg.de 